\title{
Theoretical Analysis of Two-in-one Composite 1/4 Wave-plate
}

\author{
Yu Chen ${ }^{1, a}$, Yuqing Xie ${ }^{1,2, b}$, Dong Xue $e^{1,3, c^{*}}$ \\ ${ }^{1}$ School of Opto-Electronic Engineering, Zaozhuang University, Zaozhuang277160, China \\ ${ }^{2}$ School of Mechanical and Electrical Engineering, Zaozhuang University, Zaozhuang277160, China \\ ${ }^{3}$ Zaozhuang Engineering Research Center of Terahertz, Zaozhuang 277160, China \\ a muxuchenyu@sina.com, bharrison.xie@outlook.com, ${ }^{\mathrm{b}}$ fiberlaser@126.com \\ * the corresponding author
}

Keywords: Wave-plate; Jones vector; Polarization

\begin{abstract}
The wave-plate system composed by two wave-plates of arbitrary retardation can work as a $1 / 4$ wave-plate, if the angle between the fast of axes satisfy certain relation. But common composition formula is composing of tangent function and cotangent function, which is improper for $1 / 4$ wave-plate or $1 / 2$ wave-plate. In this paper, according to the Jones vector theorems, a formula for composite 1/4 wave-plate was deduced theoretically with Jones matrix method. The solution is appropriate with simulation and experiments.
\end{abstract}

\section{Introduction}

Usual quarter-wave plate and half-wave plate are both cloven by Maria glass, but it is very difficult to clove the maria glass as strict and correct thickness [1-6]. Consequently, the research has practical significance of two-in-one composite wave-plate. When the angle between the fast of axis is a specific value, the wave-plate system composed by two wave-plates of arbitrary retardation, emergent light of the system can be elliptically polarized light though the incident light is linearly polarized light, which can replace the 1/4 wave-plate [7-11]. That literatures [12-14] have given the formula to us when a wave plate merge with another, but it hasn't explained how to use these formula. In fact, it cannot be calculated because of the limitation of value range of tangent function and cotangent function when applying $1 / 4$ wave-plate merge with $1 / 2$ wave-plate. The paper, according to the Jones vector change of incident light and emergent light, applies matrix method to derive the formula in that literature again and improve it. And it was demonstrated by detailed example how to modify it, so that applying the formula to make calculation by practice.

\section{The derivation of matrix method}

The orientation of wave-plates as shown in Figure 1, let the optical axis of Polarizer $\mathrm{P}$ on $\mathrm{X}$ axis and let it at the angel of $\theta$ to the fast-axis direction of retarder T1. At the same time, make the fast-axis direction of retarder T1 at the angel of $\alpha$ to the retarder T2.

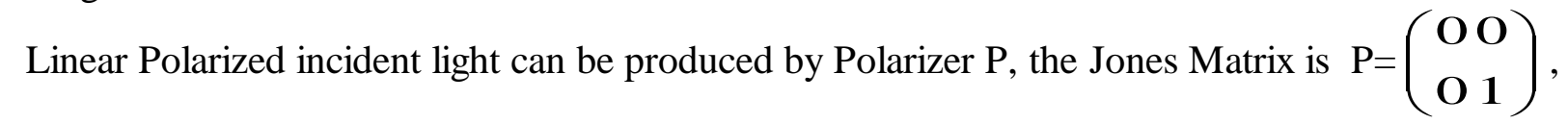
the matrix of retarder and rotation of coordinates are $\mathrm{T}(\delta)=\left(\begin{array}{cc}1 & 0 \\ 0 & e^{i \delta}\end{array}\right), \mathrm{R}(\theta)=\left(\begin{array}{cc}\cos \theta & \sin \theta \\ -\sin \theta & \cos \theta\end{array}\right)$. 


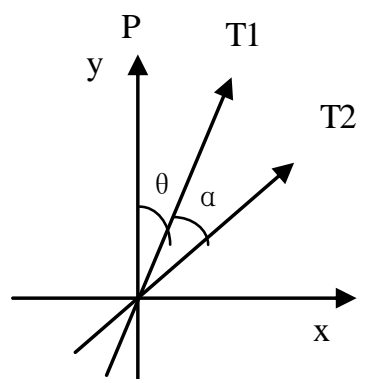

Figure.1 The orientation of wave plates

The Jones Matrix of system of two-in-one composite wave-plate (including Polarizer)

$$
\mathrm{M}=\mathrm{T}\left(\delta_{2}\right) \mathrm{R}(-\alpha) \mathrm{T}\left(\delta_{1}\right) \mathrm{R}(-\theta) \mathrm{P}=\left(\begin{array}{ll}
0 & a_{1}+i b_{1} \\
0 & a_{2}+i b_{2}
\end{array}\right)
$$

Where

$$
\begin{gathered}
\mathrm{a}_{1}=-\sin \theta \cos \alpha-\cos \theta \sin \alpha \cos \delta_{1} \\
\mathrm{~b}_{1}=-\cos \theta \sin \alpha \sin \delta_{1} \\
\mathrm{a}_{2}=\cos \theta \cos \alpha \cos \left(\delta_{1}+\delta_{2}\right)-\sin \theta \sin \alpha \cos \delta_{2} \\
\mathrm{~b}_{2}=\cos \theta \cos \alpha \sin \left(\delta_{1}+\delta_{2}\right)-\sin \theta \sin \alpha \sin \delta_{2}
\end{gathered}
$$

Given incident light's component that propagating along the $\mathrm{Z}$ axis propagating are Ex and Ey in the direction of $\mathrm{X}$ axis and $\mathrm{Y}$ axis, therefore, the Jones vector is $\mathrm{E}=\left(\begin{array}{l}E^{x} \\ E^{y}\end{array}\right)$, multiply it by $\mathrm{M}$ that can get the Jones vector of emergent light $\mathrm{E}^{\prime}=\left(\begin{array}{c}E^{x} \\ E^{y}\end{array}\right)$, let the phase angel of emergent light's component in the direction of $\mathrm{X}$ axis and $\mathrm{Y}$ axis is $\varphi_{\mathrm{x}}$ and $\varphi_{\mathrm{y}}$ it's easy to get following equation

$$
\begin{gathered}
\varphi_{\mathrm{x}}=\operatorname{atan}\left(\mathrm{b}_{1} / \mathrm{a}_{1}\right)=\operatorname{atan}\left(\frac{\cos \theta \sin \alpha \sin \delta_{1}}{\sin \theta \cos \alpha+\cos \theta \sin \alpha \cos \delta_{1}}\right) \\
\varphi_{\mathrm{y}}=\operatorname{atan}\left(\mathrm{b}_{2} / \mathrm{a}_{2}\right)=\operatorname{atan}\left[\frac{\cos \theta \cos \alpha \sin \left(\delta_{1}+\delta_{2}\right)-\sin \theta \sin \alpha \sin \delta_{2}}{\cos \theta \cos \alpha \cos \left(\delta_{1}+\delta_{2}\right)-\sin \theta \sin \alpha \cos \delta_{2}}\right]
\end{gathered}
$$

Therefore, the total phase shift that generated by two combined wave-plates is $\delta=\varphi \mathrm{x}-\varphi \mathrm{y}$

$$
=\operatorname{atan}\left[\frac{\left(\sin \delta_{1} \cos \delta_{2}+\cos \delta_{1} \sin \delta_{2} \cos 2 \alpha\right) \sin 2 \theta+\sin 2 \alpha \cos 2 \theta \sin \delta_{2}}{\left(\sin \delta_{1} \sin \delta_{2}-\cos \delta_{1} \cos \delta_{2} \cos 2 \alpha\right) \sin 2 \theta-\sin 2 \alpha \cos \theta \cos \delta_{2}}\right]
$$

above conclusions are different from that literature because of difference of direction of angle $\theta$. It can get the same result if let $-\theta=\theta$.

\section{Argumentation}

(1) Emergent light is elliptically polarized light in general.

(2) When $\delta=k \pi$, emergent light is linearly polarized light by the formula (8):

$$
\tan 2 \theta=\frac{-\sin 2 \alpha \sin \delta_{2}}{\sin \delta_{1} \cos \delta_{2}+\cos \delta_{1} \sin \delta_{2} \cos 2 \alpha}
$$

(3) When $\delta=\mathrm{k} \pi+\pi / 2$, emergent light is elliptically polarized light by the formula (5):

$$
\tan 2 \theta=\frac{\sin 2 \alpha \cos \delta_{2}}{\sin \delta_{1} \sin \delta_{2}-\cos \delta_{1} \cos \delta_{2} \cos 2 \alpha}
$$


(4) When the condition 3 and the formula $a_{12}+b_{12}=a_{22}+b_{22}$ are satisfied, it's elliptically polarized light. now $\cos 2 \alpha=\operatorname{ctg} \delta_{1} \operatorname{ctg} \delta_{2}$

\section{Application}

Above formulas need to be applied flexibly. When the angle is $\pi$ or $\pi / 2$, the formulas (5) to (8) cannot establish because of the limitation of the limitation of value range of tangent function and cotangent function. Under the circumstances, we can work from the Jones Matrix of system of two-in-one composite wave-plate and use the formulas (1) to (4) that a way safer and more convenient to solve. As an applied example, we use a 1/2 wave-plate and a $1 / 4$ wave-plate to form the two-in-one composite $\lambda / 4$ wave plate: let $\delta 1=\pi, \delta 2=\pi / 2$.

Now the angle $\alpha$ between the fast-axis cannot be found. We find that the wave plate retardation is equal to multiple another retardation. Let $\delta_{1}=2 \delta_{2}$, and plug that into the formula (8) there:

$$
\cos 2 \alpha=\frac{1-\operatorname{tg}^{2} \delta_{2}}{2 \operatorname{tg}^{2} \delta_{2}}=\frac{1}{\operatorname{tg}^{2} \delta_{2}}-\frac{1}{2}
$$

Then,if plug $\delta_{2}=\pi / 2$ into formula (9), we can get $\alpha=\pi / 3$, the same data of that literature.Plug the data into formulas(1)to(4), there:

$$
\begin{aligned}
& \mathrm{a}_{1}=-\sin \theta \cos \alpha+\cos \theta \sin \alpha=\sin (\alpha-\theta) \\
& \mathrm{b}_{2}=-\cos \theta \cos \alpha-\sin \theta \sin \alpha=-\cos (\alpha-\theta)
\end{aligned}
$$

$\mathrm{a}_{2}=\mathrm{b}_{1}=0$, only the condition $\mathrm{a}_{1}{ }^{2}+\mathrm{b}_{1}{ }^{2}=\mathrm{a}_{2}{ }^{2}+\mathrm{b}_{2}{ }^{2}$ is satisfied, emergent light is Elliptically Polarized Light, being $|\tan (\alpha-\theta)|=1$.

Therefore, $\theta=\alpha \pm \pi / 4$ and $\alpha=\pi / 3$, so $\theta=\pi / 12$ or $\theta=7 \pi / 12$. Above works explain accuracy and usage of the formula in this paper. For mica, because it's difficult to split the wave plate that has got accurate thickness we can apply above formulas to design two-in-one composite wave-plate and polarization plane rotator of every direction, which have significance to improve the accuracy of composite wave-plate.

\section{Acknowledgement}

This work is supported by national natural science fund project (No. 11447200); Shandong provincial natural science foundation (No. ZR201702200400); Science and technology program of Shandong higher education institutions (No. J17KA087); The program of independent innovation and achievement transformation plan for Zaozhuang (No. 2016GH19); Science and technology program of Zaozhuang (No. 2016GX31).

\section{References}

[1] D. Goldstein, Polarized Light, Third Edition, Crc Press, (2010).

[2] D. Clarke, J.F. Grainger, Polarized light and optical measurement, American Journal of Physics, 40 (1971) 1055-1056.

[3] J.R.M. Barr, Achromatic prism beam expanders, Optics Communications, 51 (1984) 41-46.

[4] L. Sun, Z. Lü, D. Zhang, Z. Zhao, J. Yuan, Achromatic terahertz quarter-wave retarder in reflection mode, Applied Physics B, 106 (2011) 393-398.

[5] J.R. Sambles, Polarized Light in Optics and Spectroscopy, Physics Today, 44 (1991) 117-118.

[6] R.D. Tewari, A.M. Ghodgaonkar, V. Bhattacharyya, Modified polarizing prism, Optics \& Laser Technology, 30 (1998) 63-70.

[7] S. Pancharatnam, Achromatic combinations of birefringent plates, Proceedings of the Indian Academy of Sciences - Section A, 41 (1955) 137-144.

[8] G. Ghosh, Dispersion-equation coefficients for the refractive index and birefringence of calcite and quartz crystals, Optics Communications, 163 (1999) 95-102. 
[9] J.M. Herrera-Fernandez, J.L. Vilas, L.M. Sanchez-Brea, E. Bernabeu, Design of superachromatic quarter-wave retarders in a broad spectral range, Applied Optics, 54 (2015) 9758.

[10] S.S. Ivanov, A.A. Rangelov, N.V. Vitanov, T. Peters, T. Halfmann, Highly efficient broadband conversion of light polarization by composite retarders, Journal of the Optical Society of America A Optics Image Science \& Vision, 29 (2012) 265-269.

[11] R.J. King, Quarter-wave retardation systems based on the Fresnel rhomb principle, Journal of Scientific Instruments, 43 (1966) 617.

[12] J.L. Vilas, L.M. Sanchezbrea, E. Bernabeu, Optimal achromatic wave retarders using two birefringent wave plates, Appl Opt, 52 (2013) 7081-7082.

[13] J.L. Vilas, L.M. Sanchez-Brea, E. Bernabeu, Optimal achromatic wave retarders using two birefringent wave plates: reply, Applied Optics, 52 (2013) 7081-7082.

[14] X. Zhang, Optimal achromatic wave retarders using two birefringent wave plates: comment, Applied Optics, 52 (2013) 7078-7080. 